\title{
Crisis climática y Objetivos de Desarrollo Sostenible: un enfoque desde la perspectiva de la producción animal, el consumo de carne y los efectos sociales
}

\author{
A. Guerrero ${ }^{1,2}$, J.D. Gómez-Quintero³ y J.L. Olleta2,* \\ 1 Colaboradora Cátedra Cooperación para el Desarrollo. Universidad de Zaragoza, Zaragoza, España \\ 2 Departamento Producción Animal y Ciencia de los Alimentos. Grupo acción Campus Iberus-Producción \\ de carne: biodiversidad y sostenibilidad. Universidad de Zaragoza, C/ Miguel Servet 177, 50013. Zara- \\ goza, España \\ 3 Departamento Psicología y Sociología, Universidad de Zaragoza. C/ Violante de Hungría 23, 50009. \\ Zaragoza, España
}

\section{Resumen}

El objetivo del presente trabajo es la realización de una síntesis sobre las múltiples y complejas relaciones existentes entre los Objetivos de Desarrollo Sostenible (ODS) y el ámbito de la producción animal, prestando una especial atención a sus implicaciones y consecuencias derivadas de la actual crisis climática. La Agenda 2030 para el Desarrollo Sostenible de Naciones Unidas presenta 17 ODS, que integran, de forma indivisible, las tres dimensiones del desarrollo sostenible, siendo estos pilares: el social, ambiental y económico. El sector ganadero tiene un notable rol y un significativo potencial para la implementación y consecución de dichos Objetivos, enfocadas sus posibles intervenciones desde diferentes perspectivas que se desarrollan a lo largo del presente trabajo. Entre los enfoques se abarcan las relaciones generales entre los ODS y la ganadería (especialmente centrado en rumiantes), diversas estrategias para la puesta en práctica de los ODS, el impacto de la crisis climática a nivel productivo, sus estrategias adaptativas y de mitigación. Entre los impactos sociales se identifica la movilidad humana forzada y el riesgo de hambrunas e insuficiencia alimentaria. Por último, se analiza el impacto de la producción de carne y su consumo en dietas sostenibles.

Palabras clave: ODS, ganadería, cambio climático, dietas sostenibles, impacto social.

Climatic shock and Sustainable Development Goals: an approach from the perspective of animal production, meat consumption and its social effects

Abstract

The objective of this paper is to carry out a synthesis about the multiple and complex relationships between the Sustainable Development Goals (SDGs) with the field of animal production, paying special attention to its implications and consequences derived from the current climate change.

* Autor para correspondencia: olleta@unizar.es

Cita del artículo: Guerrero A, Gómez-Quintero JD, Olleta JL (2020). Crisis climática y Objetivos de Desarrollo Sostenible: un enfoque desde la perspectiva de la producción animal, el consumo de carne y los efectos sociales. ITEA-Información Técnica Económica Agraria 116(5): 405-423. https://doi.org/10.12706/itea.2020.025 
The United Nations 2030 Agenda for Sustainable Development presents 17 SDGs, which integrate, in an indivisible way, the three dimensions of sustainable development, being these pillars: social, environmental and economic. The livestock sector has a remarkable role and significant potential for the implementation and achievement of these objectives, focusing its possible interventions from different perspectives that will be developed throughout this article. Approaches include the general relationships between the SDGs and livestock, various strategies for the implementation of the SDGs, the impact of the climate crisis on animal production and its implications at social level. As well as, aspects related to climatic migrants and food production, analyzing also the impact of meat production and its consumption on sustainable diets.

Keywords: SDGs, livestock, climate change, sustainable diet, social impact.

\section{Introducción}

\section{Principales interacciones entre ODS}

La Nueva Agenda global 2030 propuesta por la ONU entró en vigor de manera oficial el 1 de enero de 2016 y está enfocada hacia la consecución de un desarrollo sostenible. Para ello se han formulado una serie de objetivos globales cuya finalidad es erradicar la pobreza, disminuir las desigualdades y proteger el medio-ambiente, promoviendo así un desarrollo global sostenible (ONU, 2020). El reto actual internacional consiste en alcanzar las 169 metas propuestas derivadas de los 17 Objetivos de Desarrollo Sostenible (ODS) vigentes. Para ello se están implementando diferentes estrategias que guían los programas de desarrollo y las políticas mundiales y que continuarán durante la próxima década (ONU, 2019). Su consecución genera grandes desafíos por lo que necesitan una actuación conjunta implicando todos los sectores de la sociedad: gobiernos, sector público, sector privado, sociedad general, etc. fomentado, desde distintos niveles y ámbitos competenciales, políticas, actuaciones de concienciación e implementación de diferentes acciones.

Los 17 ODS integran, de forma indivisible, las tres dimensiones del desarrollo sostenible: la social, ambiental y económica. Existiendo múltiples e inseparables relaciones entre muchos de ellos. De manera práctica los ODS se podrían agrupar en aquellos que describen las necesidades y aspiraciones de los pueblos (fin de la pobreza, hambre cero, buena salud y educación, igualdad de género, reducción de las desigualdades y paz y justicia). Aquellos que describen los requisitos ambientales o "planetarios" (agua, energía, sistemas marinos, tierra y biodiversidad, y clima) y los que describen los procesos para alcanzar un mejor equilibrio entre ellos. Siendo este un enfoque pueblos-planeta-procesos (PPP) muy adecuado para sintetizar las múltiples interacciones que la ganadería presenta con los ODS (FAO, 2016). Estos fuertes vínculos y relaciones pueden ser tanto directas como indirectas asociadas a todo el sistema de agroalimentación (Campbell et al., 2018). El sector agro-ganadero tiene mucho que decir, hacer y aportar en la consecución de estos objetivos, siendo no sólo un sector productor de alimentos sino con repercusión directa en el ámbito social y medio-ambiental (Guerrero et al., 2019a). Aunque la ganadería se relaciona con los 17 ODS, existen seis más relevantes para el sector: los ODS 1 (fin pobreza), 2 (hambre cero), 12 (producción y consumo responsable), 13 (acción por el clima), 15 (vida y ecosistemas terrestres) y 17 (alianzas). La ganadería es un instrumento eficaz para la mejora de las condiciones de vida, especialmente en áreas rurales, y en regiones más desfavorecidas, ya que permite disminuir los índices de pobreza y garantizar la seguridad 
alimentaria (ONU, 2019). En los países del norte, es un sector fundamental para garantizar el abastecimiento de alimentos a la sociedad. Es una fuente de ingresos y empleo, variable según países, que representa en países de renta alta el $3 \%$ del empleo total. En países de ingreso medio estas cifras ascienden hasta el $29 \%$, y alcanzan el $60 \%$ en los de ingreso bajo (Banco Mundial, 2020). El apoyo a pequeños y medianos productores contribuye de manera significativa a reducir las desigualdades en y entre los países (FAO y AGROSAVIA, 2018). Los trabajadores de este sector fomentan la vida rural, mantienen infraestructuras en pueblos y comarcas, permiten la supervivencia poblacional en zonas de grave declive demográfico, mantienen prácticas y tradiciones que en muchos lugares del mundo constituyen parte importante del patrimonio cultural e inmaterial de la humanidad. No obstante, la caída del número de trabajadores agrícolas es vertiginosa año tras año. Según las estadísticas del Banco Mundial (2020) en 1991 el porcentaje de empleo agrícola mundial era del $44 \%$ del total del empleo mientras que en el 2018 decayó hasta el $28 \%$. En el año 1965 los países de la Unión Europea contaban con 141 millones de personas que vivían en zonas rurales. En 2016 esa cifra disminuyó hasta los 124 millones y la tendencia vigente continúa en descenso (Faostat, 2020).

La mujer desempeña un papel muy relevante, frecuentemente infravalorado en las estadísticas laborales, cuya representación oscila entre el $52 \%$ de los empleos agrarios en África del Sur, al $7 \%$ en América Latina y El Caribe o el $3 \%$ en zona Euro (Banco Mundial, 2020). Por tanto, especialmente en regiones más desfavorecidas con peor acceso a recursos, tierra y capital, priorizar la mejora de las condiciones de vida sería necesario para alcanzar la igualdad de género en el sector (FAO, 2016). El abordaje del binomio ganadería-educación incluye diversas pers- pectivas. En las regiones más desfavorecidas una mayor cantidad de ingresos derivados de la ganadería se traducen en mejores posibilidades de acceso de las familias a la educación, teniendo un considerable impacto social positivo, una mejora económica y social que favorece la reducción de desigualdades o de la brecha muchas veces existente entre poblaciones del medio rural y urbano. En regiones con más posibilidades, la educación sobre la importancia del medio rural y el origen de los distintos alimentos favorece la valorización del sector.

Es también directa la relación de la ganadería con otros ODS relacionados con el agua, con la vida en los diferentes medios (terrestres y submarinos), con los sistemas de producción y consumo responsables, así como su acción por el clima. Esta relación e impacto tiene un doble sentido de influencia. La ganadería afecta a las variaciones climáticas y medio ambientales, así como estos cambios (a los que su modificación no se debe únicamente al impacto agro-ganadero) también afectan directamente al sector. Siendo este también un instrumento indudable para generar alianzas o mediación de conflictos e integración de determinadas poblaciones, tal y como se está aplicando ya en territorios como Colombia (Zygierewicz, 2018), donde distintos proyectos de cooperación internacional en el ámbito agrario están demostrando ser una herramienta de integración efectiva.

\section{De la teoría a la práctica. Aplicación ODS en el sector bovino de carne}

El abordaje sobre los ODS es muy amplio, multidisciplinar y variado. Sin embargo, es universal que las prioridades de implementación de determinados ODS, a pesar de tener una dimensión global, variarán notablemente según las necesidades y las interacciones con los principales problemas y retos de sos- 
tenibilidad de cada región, territorio o país (Salvia et al., 2019). Las investigaciones realizadas en los últimos años (Kanter et al., 2016; Nhemachena et al., 2018; Caiado et al., 2018; Guerrero et al., 2019b) destacan algunos aspectos clave para el éxito de metas específicas de los ODS. Estas claves van desde los incentivos económicos creados por decisiones gubernamentales, la adaptabilidad a los parámetros locales, el papel de los actores implicados hasta el grado de información y conocimiento de los universitarios y profesionales especializados.

Varios informes internacionales como "2019Europe Sustainable Develpoment Report" del Instituto Europeo de Política Ambiental (SDSN e IEEP, 2019) recogen el diagnóstico de cada país, los índices e indicadores de los principales avances y retos en la consecución de los ODS, reflejándolos también de una manera gráfica mediante mapas virtuales (EU SDG Index). Otros trabajos de investigación como el de Nhemachena et al. (2018), muestran que, incluso en países pertenecientes a una misma subregión, existen peculiaridades, fortalezas y diferentes prioridades basadas en sus propias condiciones básicas.

Es fundamental poder llevar las bases teóricas a un terreno práctico y aplicativo. En esa línea, diversos proyectos dan un enfoque aplicable a las estrategias agroalimentarias disponibles para cumplir con la Nueva Agenda, esto es, "traducir los Objetivos de Desarrollo Sostenible en acción". Para ello es necesario estudiar distintos subsectores agrícolas bajo el prisma de la utilización de diversas herramientas locales y experiencias que puedan ayudar a transformar el sector agroalimentario de una manera coherente con los ODS. Como trabajo de campo concreto Kanter et al. (2016) estudiaron el sector de vacuno de carne uruguayo. En él se desarrollaron y estudiaron estrategias que permitieron un desarrollo sostenible, que maximizó la productividad, a la vez que consideró el impacto ambiental, tratando de disminuir la huella de carbono y mantener la biodiversidad, así como analizando las pérdidas de nitrógeno y fijación de carbono. Tal y como se recoge en dicho trabajo, la aplicación de este modelo desarrollado en otros países es un gran reto y cada estrategia debe adaptarse a las condiciones particulares. Ya que, en Uruguay, por ejemplo, existe una combinación de factores como incentivos económicos y ambientales que han hecho que los ODS sean una de las prioridades del gobierno. Hecho que no ocurre en otros muchos países por su situación política o ambiental. En América Latina y EI Caribe, donde el sector ganadero representa el $46 \%$ del PIB agrícola y se produce casi el $30 \%$ de la carne de vacuno mundial, las experiencias y desafíos en la producción cárnica difieren. La implementación de diferentes innovaciones que permiten una producción mediante la disminución de emisiones de Carbono varía según países y especies considerada. En la Figura 1 se observa que tanto la densidad ganadera, como el impacto de la intensidad de emisiones derivada la producción cárnica difieren considerablemente según el área geográfica. Entre las estrategias recogidas por FAO y AGROSAVIA (2018) se encuentran aquellas relacionadas con modelos de clasificación y priorización de municipios para la asignación de recursos y ejecución de proyectos sostenibles en ganadería (Brasil); impulsar sistemas de control para la producción en sistemas ecológicos (Argentina) o aplicación de nuevas metodologías para cuantificar la producción de metano entérico caracterizando la composición de la dieta, productividad y manejo de los sistemas ganaderos (Ecuador-Colombia).

En España se han desarrollado diversas iniciativas con éxito que evidencian el posible desarrollo de modelos más sostenibles aplicados a la producción de carne bovina. El proyecto "Sobrarbe Autóctono y Sostenible" desarrollado en la comarca del Sobrarbe (Huesca, Es- 


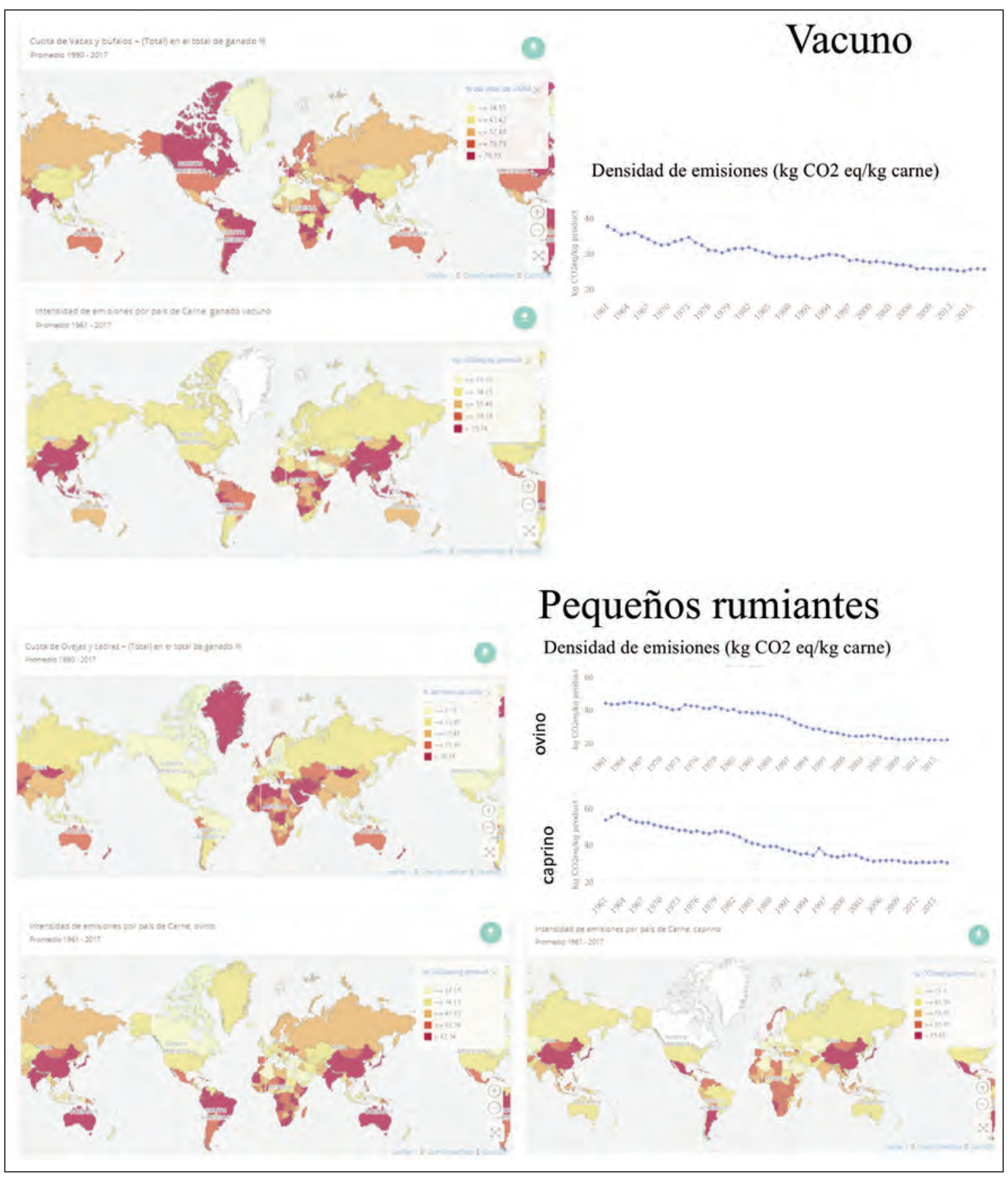

Figura 1. Distribución geográfica y su impacto en la intensidad de emisiones durante la producción de carne de rumiantes.

Fuente: Faostat (2020).

Figure 1. Geographical distribution and impact of emissions on ruminant meat production. 
paña; 2016/2018) implicó a toda la cadena de producción con una misma finalidad, la búsqueda por una producción más sostenible integral en la región. Para ello participaron conjuntamente los sectores de la Producción Animal (ganaderos de raza bovina Pirenaica), del Medio Ambiente (Fundación para la conservación del Quebrantahuesos), Administración local (ayuntamiento), proveedores de alimentos y restauración de la comarca. Algunas de las acciones más significativas desarrolladas y relacionadas con los ODS fueron:

ODS 4 (Educación) y 12 (Producción y consumo responsable):

- Promoción y difusión del concepto de la carne "de km cero" en los colegios, institutos, carnicerías y locales dedicados a restauración.

- Sensibilización a la población escolar de la importancia que tiene realizar los actos de consumo de forma consciente, crítica, sostenible, responsable y solidaria.

- Ayudar a vincular el medio rural con un mercado de productos de calidad asociado a sus materias primas de proximidad.

- Fomento de la comercialización que permita un aprovechamiento directo del productor y comprador sin la intervención de la intermediación, disminuyendo con ello los gastos de un proceso más complejo y difícil.

ODS 8 (trabajo y crecimiento) y ODS 15 (vida ecosistemas terrestres):

- Creación de las condiciones para generar empleo y el asentamiento de la población en el medio rural.

- Garantizar la continuidad de las explotaciones del sector primario como instrumento de desarrollo económico en el medio rural, vinculado a los recursos locales.

- Potenciar un comedero de aves necrófagas de la zona.
Con todas estas acciones además de colaborar con la implantación de los ODS se fomenta una economía circular donde el alimento para el mantenimiento de la especie protegida (Quebrantahuesos) procede del propio matadero comarcal. El cual presta servicio sin coste a los ganaderos de la región, con el consiguiente beneficio para la ganadería extensiva circundante y la biodiversidad. También hay un beneficio económico y medio ambiental, debido al ahorro en costes y contaminación por una menor distancia de transporte al matadero comarcal, a carnicerías de la zona, y al evitar el transporte y la incineración de cadáveres y despojos. Así como un beneficio e impacto directo sobre el eco turismo de la zona. Este comedero constituye igualmente un recurso educativo realizando visitas formativas y educativas en todos los sectores de enseñanza.

Las políticas agrarias actuales reconocen el papel fundamental de la ganadería en el mantenimiento de la diversidad ecológica, paisajística y cultural. Su multifuncionalidad productiva, medioambiental y social, así como los diversos beneficios y contribución a una economía verde que presenta la diversidad genética ganadera (Leroy et al., 2018a,b). Uno de los cambios más importantes que se ha producido en los mercados alimentarios es el paso de la venta de productos básicos, no diferenciados, a la venta de productos de calidad diferenciada (Sañudo et al., 2017). La coordinación entre los actores es más sencilla en el caso de circuitos cortos de comercialización o sistemas alimentarios locales.

El etiquetado es una herramienta importante que recoge distintos tipos de informaciones que proporcionan al consumidor la posibilidad de ser consciente y de elegir el tipo de producto que quiere comprar y consumir. Las marcas de calidad diferenciadas (Sañudo et al., 2017) o aquellas que permitan identificar un producto de cercanía, local o elaborado bajo diversos sistemas de producción específicos 
(Guerrero et al., 2019b) serían estrategias aplicables. Así por ejemplo el consumo de carne local o de razas autóctonas podría favorecer esta sostenibilidad asociado a los distintos beneficios ambientales, sociales y económicos locales que proporciona (Dietapyr2, 2020).

La Figura 2 sintetiza de una manera gráfica la relación de los conceptos abordados en el contexto de los ODS y la producción bovina. En el sector, es un desafío lograr la consecución de múltiples ODS, a la vez que incrementar la productividad agropecuaria y mejorar las condiciones de vida en áreas rurales, mientras se minimizan los efectos ambientales negativos. No existen modelos universales exportables, aunque si es genérico que para lograr el equilibrio de los ODS es necesario conocer y estudiar cada contexto de implementación. La adaptación de los modelos requiere del estudio de factores tales como parámetros locales, circunstancias específicas y actores implicados, que son determinantes en el éxito y aplicación de cada decisión o estrategia (Kanter et al., 2018).

Resulta imposible la implantación de los ODS si la información sobre los mismos no llega a los actores involucrados y estos no adquieren un compromiso para su implementación. Es evidente que no es posible implementar estrategias y actuaciones que favorezcan su consecución cuando las distintas acciones para ponerlos en marcha se desconocen (Guerrero et al., 2019b). En esta línea se encuestaron a más de 600 universitarios vinculados al sector agro-alimentario en diversos países de Iberoamérica (España, Brasil y Colombia), y se observó diferencias significativas en el grado de conocimiento de los ODS según el país, rol académico y el nivel de formación (Guerrero, 2018). Así mismo, en dicho estudio se hallaron actitudes que reflejaban una alta disposición a contribuir en la con-

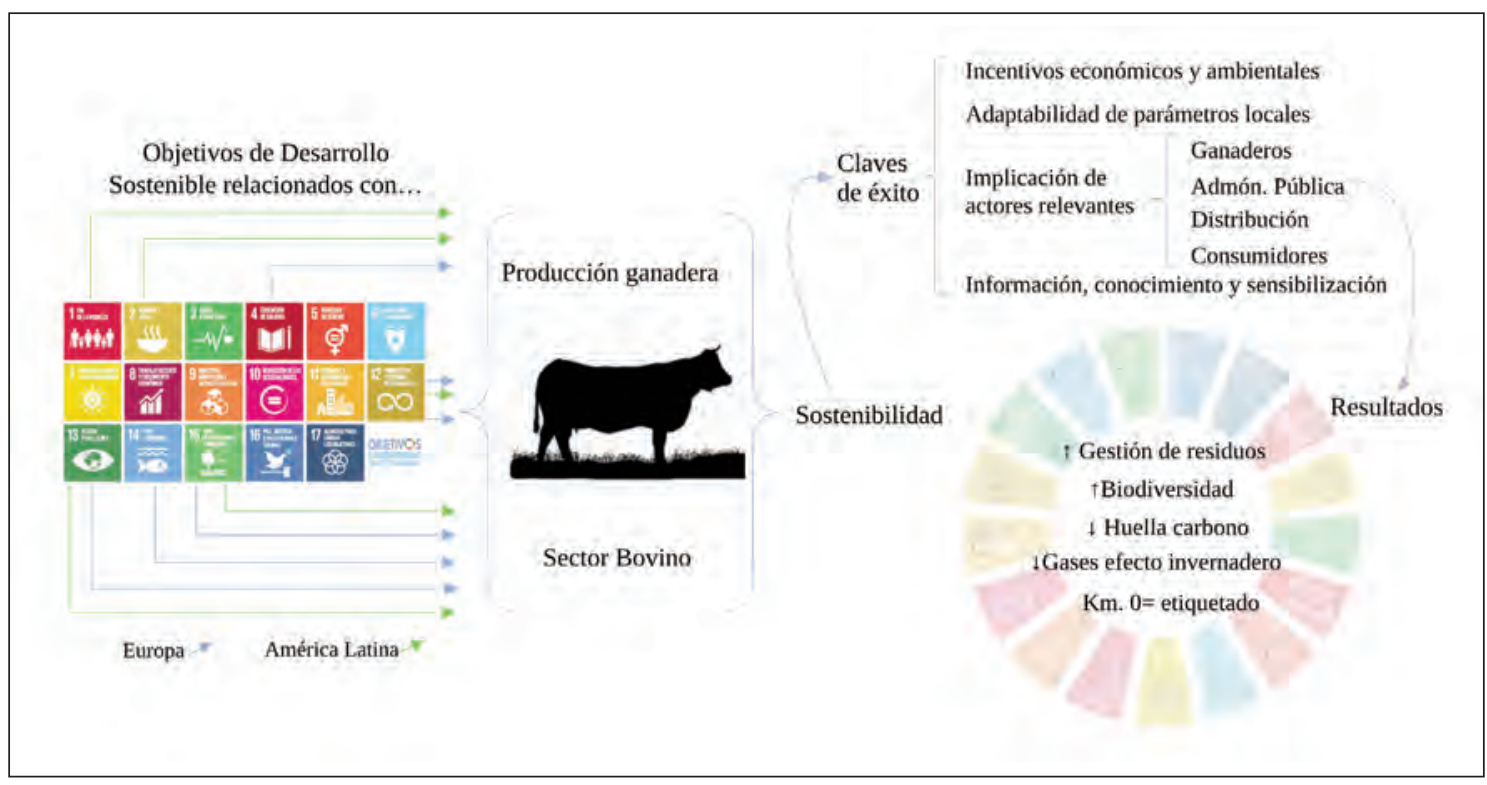

Figura 2. Relación de los objetivos de desarrollo sostenible (ODS) con la producción bovina: claves exitosas de sostenibilidad y resultados basados en evidencias.

Figure 2. Relationship between Sustainable Development Goals (SDGs) and beef production: Successful keys to sustainability and results based on evidence. 
quista de varias de las metas propuestas para alcanzar los ODS, especialmente el ODS 2 (Hambre cero) y 12 (Producción y consumo responsables). Sin embargo, a pesar de la disposición y la voluntad, muchos encuestados expresaron su desconocimiento sobre qué acciones concretas podrían realizar como agentes promotores del cambio desde su propio ámbito profesional (Guerrero et al., 2019b,c). Sería recomendable, como sugería Caiado et al. (2018) en su revisión sobre implementación de los ODS, la realización de otros estudios similares aplicados a los diversos eslabones del sector. Los que permitirían conocer las perspectivas, nivel de conocimiento e implicación con los ODS en los distintos eslabones de la cadena de producción (ganaderos, centros de procesado, intermediarios, incluso el consumidor final). Todavía son escasos los trabajos en este ámbito y serían necesarios para ayudar a comprender cómo se pueden conseguir exitosamente la implementación de los ODS en las diferentes culturas, ámbitos geográficos y sectores profesionales. Uno de los déficits más importantes en la implementación de los ODS en las regiones de renta baja es la escasa información relacionada con el diagnóstico, el seguimiento y la implementación de las metas correspondientes (Nhemachena et al., 2018). Por lo que se aboga a centrar esfuerzos futuros en la recopilación de datos para acceder a una aplicación más amplia y efectiva de los mismos que permita contribuir de una manera concreta y eficaz a la mejora de sistemas agroalimentarios sostenibles según las condiciones básicas de cada país.

\section{Crisis climática: su impacto sobre la producción animal}

Son múltiples los informes y evidencias sobre el aumento de la temperatura del último siglo, asociado a los gases de efecto inverna- dero, tendencia al calentamiento global que afectará de manera diferente a determinadas regiones, especialmente las más vulnerables (AECID, 2018; Faostat, 2020).

La producción ganadera se ve afectada directamente por las modificaciones climáticas (como se detallará posteriormente), siendo necesario el desarrollo de estrategias de adaptación. Mientras que a su vez el propio sector contribuye al cambio climático, por lo que es preciso la implementación de estrategias de mitigación para minimizar su impacto (Figura 3). El consumo de alimentos es responsable de una proporción considerable de emisiones de gases de efecto invernadero (GEI), al sector ganadero se le atribuye la responsabilidad del $14,5 \%$ de las mismas. Por tanto, se puede considerar un sector clave en la mitigación de las emisiones, así como imprescindible para garantizar la mejora y seguridad alimentaria mundial (Rojas-Downing et al., 2017), impactando directamente en los ODS.

Son múltiples los factores derivados de la crisis climática que afectan a la producción, detalladamente descritos en algunos trabajos como el de Rojas-Downing et al. (2017). Centrándonos en los rumiantes, podríamos agrupar estos factores de una manera resumida en cinco aspectos principales (Fang et al., 2015).

1. Pastos, sistemas silvopastorales. Impacto negativo sobre la biomasa de pastizales y desarrollo de modificaciones en los ecosistemas, por lo que los sistemas basados en pastoreo se ven más afectados directamente por el calentamiento global que los sistemas intensivos.

2. Los productos ganaderos. Las variaciones ambientales unidas especialmente a altas temperaturas ambientales producen efectos negativos en la producción de leche o calidad de la carne.

3. Salud del ganado. Al deterioro de la salud de los animales y disminución de su respuesta inmunológica se unen efectos di- 


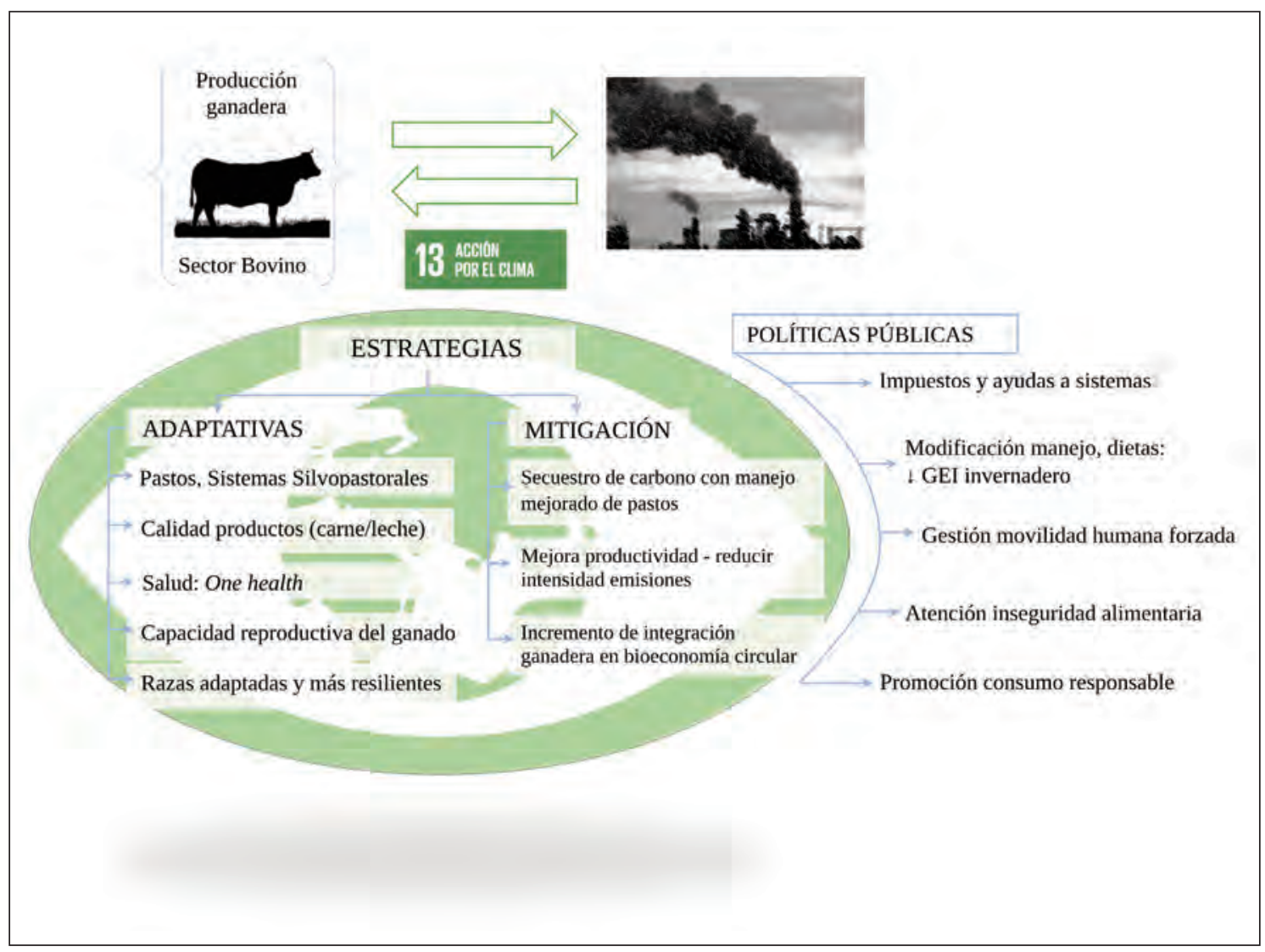

Figura 3. Estrategias de adaptación, mitigación y de acción política ante la crisis climática. Figure 3. Adaptation, mitigation and political strategies to climate crisis.

rectos de enfermedades y muertes relacionadas con la temperatura, así como variaciones en la distribución de enfermedades transmitidas por vectores y por los alimentos. Cabría remarcar en este punto la consecuencia que los aspectos relacionados con la salud animal tienen en la salud humana, siendo necesario priorizar el enfoque de Una Salud Global "One Health".

4. Capacidad reproductiva del ganado. Se ve alterada por cambios en la temperatura ambiental, diversos estudios muestran como las altas temperaturas reducen la fertilidad del ganado.
5. Impacto económico. Pérdidas en el sector de cría y producción derivadas de los distintos factores anteriormente citados.

Diferentes regiones rurales, especialmente aquellas todavía en desarrollo, donde su modo de vida está asociado a la ganadería y a los recursos extensivos, les repercute de una manera más grave las consecuencias de la crisis climática. Sufriendo una reducción y disminución de la calidad de las áreas de pastoreo, aumento de las enfermedades del ganado y escasez del agua. Factores imprescindibles en la producción ganadera que derivan en efectos devastadores a nivel socioeconómico general (Ullah et al., 
2018). La crisis climática produce oscilaciones en la distribución, en la regularidad e intensidad de las Iluvias, variación térmica, aumento de la intensidad y frecuencia de fenómenos climáticos extremos, contribuyendo a otros factores como son la pérdida de la biodiversidad y la degradación de los recursos naturales (Figura 4). Afectando su progresión de diferente manera según culturas y poblacionales, viéndose agudizado por la falta de formación o información y apoyo público sostenido en determinadas regiones (AECID, 2018).
Hace una década se publicaban trabajos cuyo objetivo era dilucidar cómo el cambio climático podría afectar a algunas variables de la producción animal poniendo como ejemplo la calidad de la carne. Gregory (2010) divide los efectos principalmente en dos. Aquellos directos derivados del estrés por calor que podrían darse en algunas especies y provocar cambios directos sobre la composición de los tejidos y el metabolismo celular, aumentando algunos defectos en la calidad de carne como DFD en rumiantes, y deshidratación en la ma-

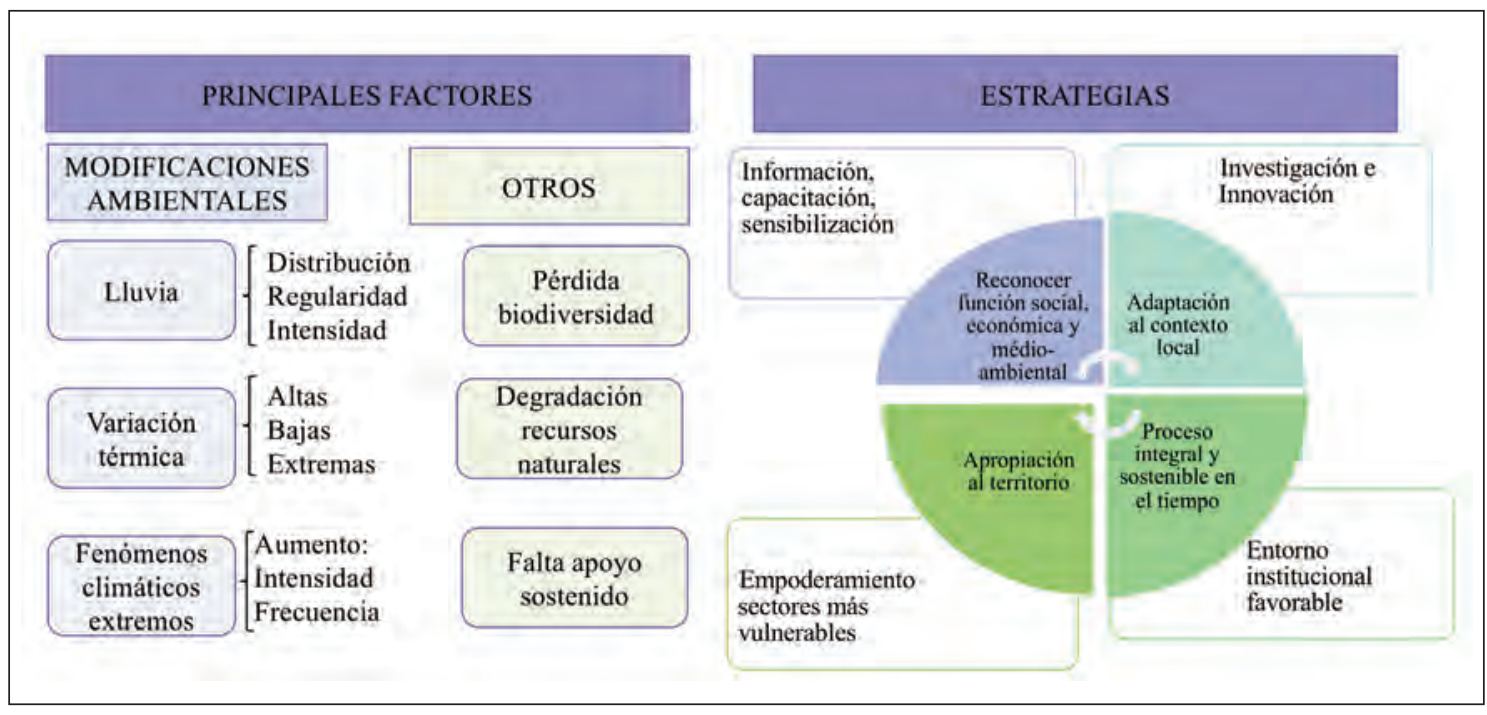

Figura 4. Principales factores que influyen en la crisis climática y estrategias para afrontarla desde los sistemas de producción ganaderos.

Fuente: Adaptado de AECID (2018).

Figure 4. Main factors influencing climate shock and strategies to deal with it from livestock production systems.

yoría de las especies. Y una segunda vertiente derivada de los cambios directos en el manejo y en las prácticas ganaderas de diversas especies. Varios estudios sobre la calidad de la carne reflejan como el sistema de producción origina modificaciones tanto en la calidad físicoquímica como organoléptica del producto, así como existen variaciones dependiendo de las propias razas (Koutsidis et al., 2008; Christensen et al., 2011; Panea et al., 2018).

Algunas de las posibles estrategias para facilitar en los animales la adaptación a las condiciones climáticas cambiantes sugeridas por Gregory (2010) fueron: Introducción y selec- 
ción de razas más tolerantes al calor; favorecer la presencia de los genotipos mejor adaptados y más resilientes. Modificaciones en el sistema de manejo, dietas y estabulación de los animales; pre acondicionamiento de estos al calor para fomentar su adaptación y mayor supervivencia ante el estrés por calor durante el transporte y procesamiento en planta.

Polli et al. (2019) recogen como las condiciones climáticas afectan mediante variaciones en los índices de temperatura y humedad, tanto al rendimiento como al comportamiento de los corderos empeorándose los resultados como consecuencia del malestar térmico. La producción lechera también se ve afectada por las variaciones de temperatura y humedad, consecuencia de los cambios climáticos durante las diferentes estaciones en las últimas décadas. Ya se están produciendo efectos adversos en la productividad lechera, y algunos escenarios predicen que el cambio climático conduciría en una reducción de entre el 5-11\% en la producción de lácteos por año en el periodo 2020-2039 (Qi et al., 2015). Existe una vulnerabilidad global en la producción mundial de alimentos ante eventos climáticos extremos que varía según la región y país analizado (Yeni y Alpas, 2017).

Parra-Cortes et al. (2019) recopilan informaciones sobre alternativas de intensificación sostenible de los sistemas de producción bovina, enfatizando en el papel que pueden tener los sistemas silvopastoriles en zonas tropicales de América Latina. Donde la integración de árboles, arbustos y razas locales serían una estrategia recomendada para la mitigación de los deterioros ambientales en dichas zonas. Ya que favorecen el mantenimiento de la diversidad, evitando la desaparición de economías familiares, incrementando los resultados productivos sin agudizar deterioros ambientales. Así como fomentan la seguridad alimentaria a partir de recursos locales, que a su vez dinamizan un desarrollo sostenible social, ambiental y económico de la región.
Existen diversas estrategias de mitigación efectivas para reducir el impacto de las industrias ganaderas y en general de todo el sector agroalimentario en el cambio climático. Como indican Hyland et al. (2017) y RojasDowning et al. (2017) estas pasan desde estrategias políticas y económicas, más relacionadas con aspectos como los impuestos y ayudas o subvenciones a determinados sistemas o sectores. Hasta otras más concretas en la propia cadena de producción, mediante implementación de estrategias que reduzcan la emisión de gases de efecto invernadero, o modificaciones de manejo, dietas, sistemas de producción y comercialización.

Una intensificación sostenible a través del aprovechamiento combinado de los diferentes recursos existentes y la aplicación de innovación e implantación de nuevas tecnologías en la alimentación y producción animal, permitirán producciones más sostenibles y respetuosas con el medio ambiente. También nuevos aditivos moduladores de la fermentación y el desarrollo de modelos de gestión más eficaces son estrategias que pueden minimizar las emisiones e impacto climático (FAO, 2019). La guía elaborada por FAO (2017) "Soluciones ganaderas para el cambio climático" propone tres formas de reducir sustancialmente emisiones de la producción ganadera mediante 1) Mejora de la productividad para reducir las intensidades de emisión; 2) Secuestro de carbono a través del manejo mejorado de pastos; 3 ) Incremento de la integración ganadera en la bioeconomía circular (Figura 3).

Por último, el comportamiento y decisión de compra de los consumidores, la elección o priorización de determinados productos tiene un gran efecto directo en los modelos de producción. Siendo conveniente para la posible realización de cambios tangibles repensar los actuales modelos de consumo y trabajar sobre la reducción del desperdicio alimentario. 
Todas las estrategias de mitigación requieren una consideración cuidadosa, estudio minucioso de todos los factores y contextualización del marco de aplicación, según las necesidades y condiciones de cada región. Como indican Campbell et al. (2018) las acciones transformadoras en el sistema alimentario para poder lograr determinados ODS, como el 13 (Acción por el clima), son cruciales, pero en todas estas acciones se deben tener en cuenta las relaciones con otros ODS.

\section{El impacto social de la crisis climática sobre la producción}

La crisis climática, en mayor o menor medida, está perjudicando directamente al entorno rural y productivo, desencadenado también una migración rural por inestabilidad de los recursos. Creándose una reubicación de diferentes especies ganaderas, debido también a la redistribución y uso de la agricultura, así como a las limitaciones del acceso a agua y/o escasez de precipitaciones. Todo ello conlleva la mudanza en la concentración de los núcleos productivos y modificaciones en la calidad de la carne y seguridad alimentaria previamente comentada (Gregory, 2010).

De acuerdo al Quinto Informe de Evaluación del Grupo Intergubernamental de Expertos sobre el Cambio Climático (IPCC, por sus siglas en inglés), hay una sólida evidencia del impacto del cambio climático sobre los sistemas naturales en todos los continentes y océanos. Los peligros vinculados a los efectos de la crisis climática afectan a las vidas de las personas pobres y vulnerables ya sea directamente, a través de impactos en los medios de subsistencia, reducciones en los rendimientos de los cultivos o a través de aumentos en los precios de los alimentos y en la inseguridad alimentaria (AECID, 2018). De este modo, nos encontramos con impactos significativos en las condiciones de vida de las poblaciones rurales y campesinas de determinadas zonas del mundo. En concreto se destacan dos consecuencias sociales, económicas y demográficas: la movilidad humana forzada y la falta de recursos alimenticios. Estas dos consecuencias suponen la dificultad para garantizar derechos sociales y económicos a las poblaciones afectadas, tales como el derecho a la vida, la salud, la educación, el trabajo, el saneamiento, la vivienda digna y el consumo de agua y otros bienes esenciales para la vida. Tal y como recoge el informe sobre los ODS 2019 (ONU, 2019), si bien los potenciales beneficios económicos de los procesos de consumo y producción sostenibles están claramente definidos, los beneficios sociales, en gran medida, todavía se pasan por alto, rara vez se miden o se incluyen en los objetivos de las normativas. Mientras el $63 \%$ de las normativas examinaron los efectos en la contaminación del aire, el suelo y el agua, o el $43 \%$ en las emisiones de gases de efecto invernadero, sólo el $11 \%$ consideró su impacto en la salud y el $7 \%$ su impacto en los géneros.

Aunque anualmente se publican cifras cada vez más fiables sobre desplazamientos internos relacionados con perturbaciones ambientales, resulta difícil obtener datos fidedignos (IDMC, 2019 en Oakes et al., 2020). Es muy complejo realizar estimaciones fiables de las personas que abandonan sus hogares en previsión o como consecuencia de procesos de evolución lenta como la desertificación, disminución de las precipitaciones o la subida del nivel del mar (IDMC, 2019 en Oakes et al., 2020).

Una de las cuestiones que sí se ha podido constatar es la tendencia creciente del desplazamiento rural-urbano que, según los contextos, se ve acelerada por la crisis climática. En los desplazamientos migratorios las mujeres son más vulnerables que los hombres porque asumen la tarea del cuidado de menores, enfermos o discapacitados durante la movilidad. Además, muchos países tienen le- 
gislaciones que dificultan el acceso a servicios y derechos de propiedad de la tierra para ellas (Resurrección et al., 2019). Esto supone el abandono de tareas productivas agrícolas, ganaderas y pesqueras realizadas en zonas rurales o pequeños núcleos, con el propósito de buscar alternativas laborales en el sector de los servicios de las grandes urbes (Oakes et al., 2020). El segundo tipo de impacto socioeconómico de la crisis climática se sitúa en el ámbito de la subalimentación y de las hambrunas ocasionadas por la escasez de recursos alimenticios y por el bajo rendimiento de los cultivos que dependen de la precipitación o que son altamente sensibles a las sequías (AECID, 2018; FAO, 2018). Las hambrunas y el déficit alimenticio no sólo son causadas por las condiciones extremas del clima, ya que también influyen los conflictos bélicos, el acceso a los insumos necesarios para la producción agrícola (semillas, fertilizantes, herramientas, etc.); pero los informes recientes (AECID, 2018; FAO et al., 2018) señalan que es uno de los factores clave del actual aumento de las crisis alimentarias graves. "El efecto acumulativo de los cambios en el clima está minando todas las dimensiones de la seguridad alimentaria, esto es, la disponibilidad de alimentos, el acceso, la utilización y la estabilidad" (FAO et al., 2018).

En concreto, en América Latina y El Caribe el impacto a medio plazo de los principales riesgos es la menor disponibilidad de agua en regiones semiáridas, el incremento de las inundaciones y los deslizamientos de tierra por precipitaciones extremas. Así como la, menor producción de alimentos y disminución de la calidad alimentaria, convirtiéndose el impacto en alto o muy alto si no se impulsan estrategias de adaptación y mitigación (AECID, 2018). Esta compleja interacción entre la crisis climática y los sistemas de producción agrícola y ganadera requiere un diseño cuidadoso y una combinación de políticas ambientales y enfoques de gestión en la producción (Figura 4). En este sentido los ODS ofrecen metas claves para el diseño de políticas y acciones claras de cara a la reorganización del manejo de las tierras, la agricultura y la ganadería, vitales para el asentamiento de la población y la eliminación del hambre.

Como refleja Fang et al. (2015), la adaptación de la producción de carne al cambio climático es un proceso continuo y dinámico. El cual hace necesaria la puesta en marcha de políticas e intervenciones efectivas que puedan mejorar la inversión en educación y capacitación en el trabajo para mejorar la productividad laboral. Políticas públicas que fomenten infraestructuras donde fuesen necesarias, proyectos de conservación de agua, subsidios de material de producción ganadera, capacitación profesional e innovación (Figura 4) son imprescindibles.

\section{¿Dietas sostenibles?}

Las dietas sostenibles son aquellas con bajo impacto ambiental que contribuyen a la seguridad alimentaria y nutricional, y a la vida sana de las generaciones presentes y futuras (FAO, 2010). Estas concurren a la protección y respeto de la biodiversidad y los ecosistemas, son culturalmente aceptables, económicamente justas, accesibles, asequibles, nutricionalmente adecuadas, inocuas y saludables, y permiten la optimización de los recursos naturales y humanos. Estando relacionadas por tanto con los múltiples ODS. De modo que las elecciones individuales que se hacen sobre este tema tienen el potencial de influir sustancialmente tanto en la salud pública, como en el medio ambiente. Y consecuentemente en todo el sector agroalimentario, con su correspondiente impacto social y económico previamente comentado.

El aporte de proteína al consumo mundial derivado de los productos ganaderos y de la carne (el cual representa el $50 \%$ de los anteriores), presenta desde la década de los 60 del 
siglo XX hasta nuestros días una tendencia creciente. Los últimos datos disponibles (Faostat, 2020) muestran como la carne representa el $17,9 \%$ de la proteína total consumida mundialmente por persona y año. Aunque tanto el consumo como la cantidad de emisión derivada de esta producción varía notablemente según el país. Especialmente basado en su nivel de ingresos, (mayores emisiones en el hemisferio Sur e importantes variaciones en el consumo según la tradición más o menos ganadera del país además del nivel de ingresos, Imagen 1). Evidenciado que los $\mathrm{kg} \mathrm{CO}_{2}$ eq. por kg de proteína, podrían reducirse notablemente mediante inversión en mejoras de la eficiencia de los sistemas ganaderos. Las especies de rumiantes suelen emitir muchos más gases de efecto invernadero por unidad de proteína en comparación con el monogástrico, siendo la media entre 201 y 295 en pequeños rumiantes y vacuno, frente 55 y 35 $\mathrm{kg} \mathrm{CO}_{2}$ eq. por kg de proteína para los suinos o aves respectivamente. El metano entérico representa más de la mitad de emisiones de gases de efecto invernadero en rumiantes, mientras que la producción de piensos, uso del suelo y el manejo del estiércol son los principales contribuyentes a las emisiones en sistemas de monogástrico (FAO, 2019). Aunque es constante encontrar alegatos enfocados en mostrar la insostenibilidad de las dietas actuales y reducción del consumo de carne, ha quedado demostrado en los inciertos tiempos actuales azotados por pandemias internacionales, que la ganadería es un sector esencial, que ha mantenido constante su actividad para poder garantizar el suministro de alimentos a toda la población. Y a su vez, durante el confinamiento se ha producido un drástico descenso y mejora de la calidad del aire como han reflejado las distintas imágenes satélites de la Agencia Espacial Europea, asociado a la paralización de otros sectores como la reducción de la actividad industrial y transporte.

Revisiones como la de Hyland et al. (2017) recogen la complejidad de la relación entre consumo de alimentos (haciendo hincapié en el consumo de carne) y su relación con el medio-ambiente y cambio climático, analizando posibles estrategias que podrían implementarse para mitigar su impacto climático. Las cuales pasan por un ajuste adaptado según las necesidades poblacionales y la ubicación de los sistemas de producción ganadera en uso (por ejemplo, en cada tipo de carne el eslabón donde actuar para reducir las emisiones varía), unido al apoyo de políticas que faciliten la implementación de estas medidas de adaptación y mitigación del cambio climático (Rojas-Downing et al., 2017).

Una dieta se considerará sostenible si se adhiere a los principios anteriormente descritos por la FAO. Sin embargo, las dietas se componen de agregados de alimentos individuales, por lo que la reducción en el consumo de carne no necesariamente conduce a reducciones en las emisiones generales e incluso puede aumentar las emisiones de gases de efecto invernadero en la dieta dependiendo de los alimentos que se usan para reemplazarlo. Distintos autores sugieren la nula necesidad de evitar categorías enteras de alimentos para poder adherirse al concepto de sostenibilidad alimentaria (Hyland et al., 2017).

La realización de estudios más precisos y actuales sobre la composición nutricional de la carne (ya que se ha demostrado las grandes diferencias entre tipos de animales en función de sus características como edad, aptitud, sistema de alimentación, etc.) permitirán reanalizar y replantear los requerimientos, necesidades y pautas de consumo, con la generación de tablas nutricionales actualizadas que permitan la elaboración de dietas más reales, completas, variadas y sostenibles. Diversos estudios como Mesana et al. (2013) han demostrado como el consumo regular de otras carnes rojas como la carne de cordero puede formar parte de una dieta sana, variada y bien equilibrada. Por tanto, el siguiente paso sería intentar compaginar simultánea- 
mente las directrices dietéticas recomendadas (con sus revisiones por la disponibilidad de continuos nuevos datos) con la reducción de las emisiones derivadas de la producción de los alimentos incluidos en ellas.

La educación y transferencias al consumidor sobre las características nutricionales, beneficios y posibles riesgos asociados a distintos tipos de carne (McAfee et al., 2010; Pereira y Vicente, 2013) son esenciales para poder escoger una alimentación sana, desde un punto de vista crítico y responsable. Existen plataformas que aúnan conceptos entre los binomios (carne y salud o carne y ciencia) donde se dispone de una manera divulgativa diversos tipos de informaciones y estudios que muestran la otra cara del consumo de carne (Carne y salud, 2019).

Otro aspecto frecuentemente cuestionado es la accesibilidad por precio a determinados tipos de alimentos, entre ellos la carne bovina. En la Tabla 1 se recoge una comparativa sobre los precios de diferentes tipos de alimentos incluidos en una dieta y su precio en España tanto por kilogramo, como por kilogramo de proteína o hierro que aportan, así como los kilogramos de consumo de los diferentes productos que serían necesarios para determinadas ingestas de nutrientes. En dicha Tabla se observa cómo el pan, un alimento básico en nuestra dieta mediterránea es altamente competitivo y la carne bovina, tantas veces criticada, no está tan mal posicionada en precio como inicialmente se piensa respecto a los vegetales. La carne roja está frecuentemente estigmatizada y discurre entre diversas estrategias para la reducción de las emisiones de carbono. Sin embargo, cabe remarcar que es la única fuente dietética de ciertos nutrientes esenciales; por lo tanto, eliminarla de la dieta podría presentar desafíos de salud (Binnie et al., 2014), así como consecuencias económicas, sociales y medio ambientales derivadas de su sustitución por otro tipo de alimentos. Por lo que

Tabla 1. Comparativa del aporte nutricional de diversos productos y precios.

Table 1. Comparison of various products (nutritional contribution and prices).

\begin{tabular}{lcccc}
\hline & $€ / \mathrm{kg}$ & $€ /$ kg proteína & $€ / \mathrm{kg}$ hierro & kg consumidos/kg proteína \\
\hline Pan blanco & 2,45 & 29,8 & 942 & 12,2 \\
Leche fresca & 0,98 & 31,6 & 10.888 & 32,2 \\
Lechuga & 5,05 & 360,7 & 6.312 & 71,0 \\
Naranja & 1,64 & 164,0 & 32.800 & 99,0 \\
Carne bovina & 12,4 & 60,7 & 4.960 & 4,9 \\
\hline
\end{tabular}

Fuente: Sañudo (2019).

cualquier herramienta de política alimentaria desarrollada para aunar salud y sostenibilidad debería ser abordado desde un foco holístico, en lugar de concentrarse únicamente en un determinado grupo de alimentos. Siendo imprescindible enfatizar sobre las consecuencias que tiene en general un sobre-consumo de alimentos. El cual es un aspecto del discurso de la dieta sostenible al que se le presta poca atención, pero con grandes implicaciones significativas, no sólo abordables desde la variable relacionada con la 
sostenibilidad y emisión prescindible de gases, sino también desde la perspectiva de salud pública y la creciente epidemia mundial de obesidad (Hyland et al., 2017; FAO, 2019; ONU, 2019). A esta disminución de consumo o consumo más racional, sin sobrepasar los requerimientos individuales, se le puede sumar otra estrategia efectiva y posible de implementar en todos los sectores, estando relacionada con la "reducción". Disminución tanto de los residuos, mediante un mayor aprovechamiento integral, circular de todos los productos o subproductos, así como la reducción a nivel individual del desperdicio alimentario, por todo el gasto energético y coste medio ambiental derivado que supone el desechar por distintos motivos cualquier alimento de consumo tanto humano como animal.

\section{Conclusiones}

La interacción entre los Objetivos de Desarrollo Sostenible, producción animal y crisis climática es un hecho palpable que requiere la aplicación de diferentes estrategias, tanto de adaptación de los sistemas y animales al medio actual cambiante, como de mitigación, para reducir su efecto sobre el medio ambiente en relación con la emisión de gases o modificaciones del uso de la tierra.

El enfoque, abordaje e implementación de las estrategias aplicables para la implementación de los ODS dependen de los factores y prioridades de cada país o región a considerar. Pero es común la necesidad de una mayor concienciación de todos los eslabones (desde el productor al consumidor), investigación, innovación y apoyo público sostenido.

La crisis climática tiene consecuencias sociales tangibles, generalmente poco visibilizadas o estudiadas que además de repercutir en la producción, afectan a la consecución de otros ODS prioritarios ligados indirectamente a la ganadería (ODS 2, 4, 12, 13, 17).
La producción animal puede ser un instrumento efectivo que ayude a canalizar la emergencia climática e implementación de los ODS, colaborando en la consecución de un desarrollo sostenible a nivel medioambiental, social y económico. Pero para ello se requiere todavía de la aplicación de innovaciones técnica y estudios que permitan una producción más eficiente y responsable. Así como replantearse y adecuar los modelos actuales de producción y consumo, donde las dietas se formulen desde una perspectiva holística, en la que también la carne pueda colaborar en su aportación tanto nutricional como social y cabida dentro de un modelo de dieta sostenible.

\section{Referencias bibliográficas}

AECID (2018). Cultivando resilencia frente al cambio climático: lecciones aprendidas para contribuir a la seguridad alimentaria y al derecho a la alimentación en América Latina y el Caribe. Segura AR, Morán S (Coord.). Agencia Española de Cooperación Internacional para el Desarrollo, Madrid. Disponible en: http://www.aecid.es/Centro-Documentacion/Documentos/Publi caciones\%20AECID/Guia\%20did\%C3\%A1ctica_ rev.pdf (Consultado: 20 enero 2020)

Banco mundial (2020). Estadísticas disponibles en: https://datos.bancomundial.org/ (Consultado: 18 febrero 2020).

Binnie MN, Barlow K, Johnson V, Harrison C (2014). Red meats: Time for a paradigm shift in dietary advice. Meat Science 98: 445-451. https://doi.org/ 10.1016/j.meatsci.2014.06.024

Caiado RGG, Leal Filho W, Quelhas OLG, Nascimento DLM, Ávila LV (2018). A Literature-based review on potentials and constraints in the implementation of the sustainable development goals. Journal of Cleaner Production 198: 1276-1288. https://doi.org/10.1016/j.jclepro.2018.07.102

Campbell BM, Hansen J, Rioux J, Stirling CM, Twomlow S, Wollenber EL (2018). Urgent action to combat climate change and its impacts (SDG 13): transforming agriculture and food systems. 
Current Opinion in Environmental Sustainability 34: 13-20. https://doi.org/10.1016/j.cosust. 2018.06.005

Carne y salud (2019). Carne y ciencia. Disponible en: https://www.carneysalud.com/carne-y-ciencia/ (Consultado: 18 diciembre 2019).

Christensen $M$, Ertbjerg $P$, Failla $S$, Sañudo $C$, Richardson RI, Nute G, Olleta JL, Panea B, Albertí P, Juárez M, Hocquette JF, Williams JL (2011). Relationship between collagen characteristics, lipid content and raw and cooked texture of meat from young bulls of fifteen European breeds. Meat Science 87: 61-65. https://doi.org/ 10.1016/j.meatsci.2010.09.003

Dietapyr2 (2020). Diez Razones para Consumir carne. Disponible en: http://dietapyr2.com/es/resultados.html (Consultado: 22 marzo 2020).

Fang YP, Liu YW, Yan X (2015). Meat production' sensitivity and adaptation to precipitation concentration index during the growing season of grassland: Insights from rural households. Agricultural and Forest Meteorology 201: 5160. http://dx.doi.org/10.1016/j.agrformet.2014. 11.001.

FAO (2010). Sustainable Diets and Biodiversity: Directions and Solutions for Policy, Research and Action. International Scientific Symposium, Biodiversity and Sustainable Diets United Against Hunger, FAO Headquarters, Rome, Italy, 3-5 November 2010. 307 pp.

FAO (2016). Síntesis - Ganadería y los Objetivos de Desarrollo Sostenible. Programa Mundial de Ganadería Sostenible. AGAL Livestock Information, Sector Analysis and Policy Branch.

FAO (2017). Livestock solutions for climate change.

FAO, FIDA, OMS, PMA, UNICEF (2018). El estado de la seguridad alimentaria y la nutrición en el mundo 2018. Fomentando la resiliencia climática en aras de la seguridad alimentaria y la nutrición. Roma, FAO. 199 pp.

FAO y AGROSAVIA (2018). Innovaciones en producción cárnica con bajas emisiones de carbono. Experiencias y desafíos en ALC.

FAO (2019). Five practical actions towards low-carbon livestock. Rome.
FAOSTAT (2020). Food and Agriculture Organization statistical database. Disponible en: http:// www.fao.org/faostat/es (Consultado: 01 marzo 2020).

Gregory NG (2010). How climatic changes could affect meat quality. Food Research International 43: 1866-1873. https://doi.org/10.1016/j.foodres.2009.05.018

Guerrero A (2018). Información y compromiso de los ODS 2 (hambre cero) y 12 (producción y consumo responsables) entre universitarios iberoamericanos del sector agroalimentario. TFM; Facultad de Economía y Empresa. Master propio en cooperación para el desarrollo. Universidad de Zaragoza. Septiembre 2018.

Guerrero A, Gómez-Quintero JD, López O (2019a). Los Objetivos de Desarrollo Sostenible (ODS) en el mundo agroganadero. Boletín Agro-Ganadero 144: 14-05-2019.

Guerrero A, Sañudo C, Olleta JL, López O, GómezQuintero JD (2019b). Conocimiento y aplicabilidad de las marcas de calidad como estrategia de implementación de los objetivos de desarrollo sostenible: estudio sobre la comunidad universitaria iberoamericana del sector agroalimentario. I Congreso Iberoamericano de Marcas de Calidad de Carne y de Productos Cárnicos, 24 y 25 octubre, Bragança, Portugal, pp. 29-33.

Guerrero A, Gómez-Quintero JD, López O (2019c) Estrategias y acciones aplicables en el sector ganadero y agrario para favorecer la implementación de los ODS. Boletín Agro Ganadero 145: 21-05-2019.

Hyland JJ, Henchion M, McCarthy M, McCarthy SN (2017). The role of meat in strategies to achieve a sustainable diet lower in greenhouse gas emissions: A review. Meat Science 132: 189-195. http://dx.doi.org/10.1016/j.meatsci.2017.04.014

IDMC (2019) Centro de Seguimiento de los Desplazamientos Internos. Global Report on Internal Displacement 2019. IDMC, Ginebra.

SDSN, IEEP (2019). The 2019 Europe sustainable development report. Sustainable Development Solutions Network and Institute for European Environmental Policy. Paris y Bruselas. Disponible en: https://eu-dashboards.sdgindex.org/ (Consultado: 25 enero 2020). 
Kanter DR, Schwoob MH, Baethgen WE, BervejiIlo JE, Carriquiry M, Dobermann A, Ferraro B, Lanfranco B, Mondelli M, Penengo C, Saldias R, Silva ME, Soares de Lima JM (2016). Translating the sustainable development goals into action: A participatory backcasting approach for developing national agricultural transformation pathways. Global Food Security 10: 71-79. http://dx.doi.org/10.1016/j.gfs.2016.08.002

Kanter DR, Musumba M, Wood SLR, Palm C, Antle J, Balvanera $\mathrm{P}$, Dale VH, Havlik $\mathrm{P}, \mathrm{Kline} \mathrm{KL}$, Scholes RJ, Thornton P, Tittonell P, Andelman S (2018). Evaluating agricultural trade-offs in the age of sustainable development. Review. Agricultural Systems 163: 73-88. http://dx.doi.org/ 10.1016/j.agsy.2016.09.010.

Koutsidis G, Elmore JS, Oruna-Concha MJ, Campo MM, Wood JD, Mottram DS (2008). Water-soluble precursors of beef flavour. I. Effect of diet and breed. Meat Science 79: 124-130. https:// doi.org/10.1016/j.meatsci.2007.08.008

Leroy G, Baumung R, Boettcher P, Besbes B, From T, Hoffmann I (2018a) Animal genetic resources diversity and ecosystem services. Global Food Security 17: 84-91. https://doi.org/10.1016/j.gfs. 2018.04.003

Leroy G, Hoffmann I, From T, Hiemstra SJ, Gandini G (2018b) Perception of livestock ecosystem services in grazing areas. Animal 12: 2627-2638. https://doi.org/10.1017/S1751731118001027

McAfee AJ, McSorley EM, Cuskelly GJ, Moss BW, Wallace JMW, Bonham MP, Fearon AM (2010). Red meat consumption: An overview of the risks and benefits. Review. Meat Science 84: 113. https://doi.org/10.1016/j.meatsci.2009.08.029

Mesana MI, Santaliestra AM, Fleta J, Campo MM, Sañudo C, Valbuena I, Martínez P, Horno J, Moreno LA (2013) Changes in body composition and cardiovascular risk indicators in healthy Spanish adolescents after lamb- (Ternasco de Aragón) or chicken-based diets. Nutrición Hospitalaria 28: 726-733. https://doi.org/10.3305/ nh.2013.28.3.6382

Nhemachena C, Matchaya G, Nhemachena CR, Karauaihe S, Muchara B, Nhlengethwa S (2018). Measuring baseline agriculture-related sustai- nable development goals index for Southern Africa. Sustainability 10: 849. https://doi.org/ 10.3390/su10030849

Oakes R, Banerjee S, Warner K (2020) Cap 9. Movilidad humana y adaptación al cambio ambiental. En: Informe sobre las migraciones en el mundo 2020 (Ed. International Organization for Migration) pp. 253-284.

ONU (2019). Informe de los Objetivos de DesarroIlo Sostenible 2019. Naciones Unidas, Nueva York, EEUU.

ONU (2020). ODS. Objetivos de Desarrollo Sostenible. Disponible en: http://www.un.org/sustainabledevelopment/es/objetivos-de-desarrollosostenible/ (Consultado: 30 mayo 2020).

Panea B, Olleta JL, Sañudo C, Campo MM, Oliver MA, Gispert M, Serra X, Renand G, Oliván MC, Jabet S, García S, López M, Izquierdo M, GarcíaCachán MD, Quintanilla R, Piedrafita J (2018). Effects of breed-production system on collagen, textural, and sensory traits of 10 European beef cattle breeds. Journal of Texture Studies 49: 528-535. https://doi.org/10.1111/jtxs.12350

Parra-Cortes RI, Magaña-Magaña MA, Piñeiro-Vázquez AT (2019). Intensificación sostenible de la ganadería bovina tropical basada en recursos locales: alternativa de mitigación ambiental para América Latina. Revisión Bibliográfica ITEA-Información Técnica Económica Agraria 115: 342359. https://doi.org/10.12706/itea.2019.003

Pereira MCC, Vicente AFRB (2013). Meat nutritional composition and nutritive role in the human diet. Meat Science 93: 586-592. https://doi.org/ 10.1016/j.meatsci.2012.09.018

Polli VA, Vaz RZ, Carvalho S, Costa PT, Mello RO, Restle J, Nigeliskii AF, Silveira IDB, Pissinin D (2019). Thermal comfort and performance of feedlot lambs finished in two climatic conditions. Small Ruminant Research 174: 163-169. https://doi.org/10.1016/j.smallrumres.2019. 03.002

Resurrección BP, Goodrich CG, Song Y, Bastola A, Prakash A, Joshi D, Liebrand J, Shah SA (2019). In the shadows of the Himalayan mountains: Persistent gender and social exclusion development. En: The Hindu Kush Himalaya Assessment (Eds. P. Wester, A. Mishra, A. Mukherji y A. Bhakta 
Shrestha), pp 491-516. Springer, Londres. https:// doi.org/10.1007/978-3-319-92288-1_14

Rojas-Downing MM, Nejadhashemi AP, Harrigan T, Woznicki SA (2017). Climate change and livestock: Impacts, adaptation, and Mitigation. Climate Risk Management 16: 145-163. http:// dx.doi.org/10.1016/j.crm.2017.02.001

Qi L, Bravo-Ureta BE, Cabrera VE (2015). From cold to hot: Climatic effects and productivity in Wisconsin dairy farms. Journal Dairy Science 98: 8664-8677. http://dx.doi.org/10.3168/jds. 2015-9536

Salvia AL, Filho WL, Brandli LL, Griebeler JS (2019). Assessing research trends related to Sustainable Development Goals: local and global issues. Journal of Cleaner Production 208: 841-849. https://doi.org/10.1016/j.jclepro.2018.09.242

Sañudo C, Guerrero A, Magalhaes D, Campo MM (2017) Importancia y necesidades de las marcas de calidad en la carne y productos cárnicos (pasado, presente y futuro). En: Guía Práctica Marcas de Calidad y Productos Cárnicos (Ed. Teixeira A, Red CYTED 116RT503, Marcarne), pp. 5-15. Instituto Politécnico de Bragança, Portugal.
Sañudo C (2019) Conferencia Magistral: Reflexiones sobre la carne y su filosofía. Congreso lberoamericano de marcas de Calidad de Carne y Productos Cárnicos. 24 y 25 octubre, Bragança, Portugal.

Ullah H, Rashid A, Liu G, Hussain M (2018). Perceptions of mountainous people on climate change, livelihood practices and climatic shocks: A case study of Swat District, Pakistan. Urban Climate 26: 244-257. https://doi.org/10.1016/ j.uclim.2018.10.003

Yeni F, Alpas H (2017). Vulnerability of global food production to extreme climatic events. Food Research International 96: 27-39. http://dx.doi. org/10.1016/j.foodres.2017.03.020

Zygierewicz A (2018) Acuerdo comercial entre la Unión Europea y Colombia y Perú. Evaluación europea de la aplicación. Ed.EPRS, Servicio de Estudios del Parlamento Europeo. https://doi. org/10.2861/630919

(Aceptado para publicación el 23 de julio de 2020) 